\title{
Home Visiting Services During the COVID-19 Pandemic: Program Activity Analysis for Family Connects
}

\author{
Anna Rybińska ${ }^{1} \oplus \cdot$ Debra L. Best $^{2} \cdot$ W. Benjamin Goodman ${ }^{1} \cdot$ Winona Weindling ${ }^{1} \cdot$ Kenneth A. Dodge $^{3}$
}

Accepted: 26 November 2021 / Published online: 10 January 2022

(c) The Author(s), under exclusive licence to Springer Science+Business Media, LLC, part of Springer Nature 2021

\begin{abstract}
Purpose Early reports highlighted challenges in delivering home visiting programs virtually during the COVID-19 pandemic but the extent of the changes in program implementation and their implications remains unknown. We examine program activity and families' perceptions of virtual home visiting during the first nine months of the pandemic using implementation data for Family Connects (FC), an evidence-based and MIECHV-eligible, postpartum nurse home visiting program.

Description Aggregate program implementation data for five FC sites for January-November of 2019 and 2020 are compared. The COVID-19 Modification Survey is used to analyze families' reactions to virtual program delivery.

Assessment Post-pandemic onset, FC's program completion rates amounted to $86 \%$ of the pre-pandemic activity level. Activity in key components of the intervention-home-visitor education and referrals to community agencies-was maintained at $98 \%$ and $87 \%$ of the pre-pandemic level respectively. However, education and referrals rates declined among families of color and low-income families. Finally, families reported a positive response to the program, with declines in feelings of isolation and increases in positive attitudes toward in-person medical care-seeking due to FC visits.

Conclusions During the first nine months of the COVID-19 pandemic, families' interest in home visiting remained strong, performance metrics were maintained at high levels, and families responded positively to the virtual delivery of home visiting. Home visiting programs should continue implementation with virtual modifications during the remainder of the pandemic but attention is needed to address growing disparities in access to home visiting benefits among marginalized communities. COVID-19 pandemic · Family Connects · MIECHV · Virtual home visiting
\end{abstract}

Anna Rybińska

a.rybinska@duke.edu

Debra L. Best

debra.best@duke.edu

W. Benjamin Goodman

ben.goodman@duke.edu

Winona Weindling

winona.weindling@duke.edu

Kenneth A. Dodge

dodge@duke.edu

1 Center for Child and Family Policy, Duke University, Duke, Box 90539, Durham, NC 27708, USA

2 Department of Pediatrics, Duke University School of Medicine, UMC, Box 3675, Durham, NC 27710, USA

3 Sanford School of Public Policy, Duke University, Box 90245, Durham, NC 27708, USA

\section{Significance}

Home visiting programs temporarily transitioned to providing services virtually when the Covid-19 pandemic started. Reports about home-visiting program activity using virtual means post-pandemic onset remain scarce and families' perceptions of virtual home-visiting services remain understudied. In this paper, we compare program activity for a universal postpartum home-visiting program (Family Connects) between pre-pandemic in-person services and post-pandemic onset virtual services. Families' reactions to virtual home-visiting services are also described. Findings can be used to inform maternal, infant, and early childhood home visiting programs' implementation during the continuing public health emergency to support program staff and participating families. 


\section{Introduction}

Home visiting programs are an established public health service designed to promote maternal and infant health and family well-being. In the United States, \$400 million in federal funds are allocated annually to evidence-based home visiting programs through the Maternal, Infant, and Early Childhood Home Visiting Program (MIECHV) (Health Resources \& Services Administration, 2020). In 2019 alone, 1,540,000 American parents and children participated in visiting programs supported by MIECHV (Health Resources \& Services, Administration, 2020).

During the ongoing COVID-19 pandemic, most home visiting models transitioned from in-home visits to virtual visiting (Zero To Three, 2020) and faced a daunting challenge of delivering care and intervention without in-home presence. The shift generated questions about the feasibility of delivering federally-funded programs via virtual means and the appropriateness of using federal and local funds to support home visiting services which are delivered virtually rather than in-person. At the same time, families with small children have been severely affected by unemployment, lack of childcare, and isolation from extended family during the pandemic (Cluver et al., 2020). In these unprecedented times, virtual home visiting services constitute an essential connection for families, addressing immediate needs and connecting families to community agencies for further support (Williams et al., 2020).

How has home visiting fared during the pandemic? Early evidence about the impact of COVID-19 on home visiting comes from home visiting staffs' self-reports collected in spring and summer of 2020 (Marshall et al., 2020; SelfBrown et al., 2020). These findings indicate that home visiting programs remained operational during the pandemic, but performance was affected by challenges in virtual program delivery (for instance, limited internet connectivity or lack of adequate devices to participate in telehealth) and COVID19 related disruptions in providers' and families' daily routines (such as lack of adequate child care or need to home school) (Marshall et al., 2020; Self-Brown et al., 2020).

What remains unknown is the impact of virtual delivery and the pandemic on home visiting program performance metrics such as program uptake, follow-through rates, and adherence to evidence-based program components such as community referrals. In addition, little is known about families' perception of receiving home visiting via virtual means during the pandemic. We address these questions by examining program activity during the pandemic for Family Connects (FC), a MIECHV-eligible, brief postnatal nurse home visiting program.

Additionally, research is needed to analyze home visiting program activity among marginalized populations in the United States post-pandemic onset. Populations with existing vulnerabilities and inequitable access to resources and health care constitute a large proportion of home visiting programs' participants (Health Resources \& Services Administration, 2016) and greatly benefit from home visiting services (Administration for Children and Families, 2020). Because marginalized communities are disproportionately affected by the pandemic (Kirby, 2020; Raifman \& Raifman, 2020), home visiting programs are uniquely positioned to provide crucial support for vulnerable families in times of unprecedented hardship if equitable access to home visiting interventions and their key aspects is maintained. In this paper, we present FC program activity across racial and ethnic and social class lines with respect to evidencebased program components of nurse education provision and referral rates.

\section{Family Connects Model and Its Modifications During Covid-19}

FC is a universal nurse home visiting program first implemented in Durham, North Carolina in 2009 and currently serving families in 23 communities across the United States (Family Connects International, 2019a, 2019b). Under the FC protocol, families residing in participating communities are invited to participate in the program shortly after birth. Enrolled families receive an integrated home visit (IHV) from a registered nurse about three weeks after the delivery. During the visit, family strengths and needs in four domains: health care, infant care, home safety, and parental well-being are assessed. When needs are identified, the nurse provides education and supportive guidance and-if need for longterm support is identified-connects the family with community resources. Nurses may also offer one to two followup home visits or phone calls for continued assessment and intervention, based on clinical judgment. Four weeks after the IHV, a FC team member follows up with a post-visit connection call to assess family satisfaction and confirm successful connections with community resources. Findings from two randomized controlled trials of FC have shown high participation rates, strong connections to community resources, high family satisfaction, and positive impact on reducing maternal mental health problems, infant emergency medical care costs, and Child Protective Services investigations (Alonso-Marsden et al., 2013; Dodge et al., 2013, 2014, 2019; Goodman et al., 2019, 2021).

FC mandated all sites transition to provide services to families via virtual means on March 18, 2020. Within the modified virtual protocol, FC sites offer two approaches to substitute the traditional in-home visit: (1) a modified IHV or (2) a structured supportive call. During the modified IHV, the nurse follows the standard IHV procedure, but the physical assessment of the caregiver and the infant have 
been replaced by detailed questions about caregiver's and infant's health. The nurse also provides guidance across the domains indicated in the standard IHV. The supportive call is a shorter intervention which simulates the IHV protocol. The caregiver is asked about feeding, mood, healthcare access, concerns, and need for follow-up. Brief education is delivered with specific attention to postpartum warning signs. The call ends with a summary of family strengths, a review of recommendations, and a plan for follow-up. Postpandemic onset, the follow-up protocol still includes one to two phone calls based on the nurse's judgement and a final connection call at four weeks post-visit.

\section{Data and Methods}

Research protocols for this study were approved by the Duke University Health Systems IRB (Protocol \#00105777) and Duke University Campus IRB (Protocol \#2021-0197). The study received an IRB waiver of informed consent. Two data sources are used: the FC program activity data and the COVID-19 Modification Survey. We selected FC program activity data from March to November in 2019 and 2020 for five certified and mature sites $(\mathrm{N}=7791$ scheduled visits). Mature sites are defined as sites with over 18 months of activity before January 2020 and demonstrated program fidelity. Our selection of mature FC sites for the program performance analysis assures that no changes or expansion of the program in the respective areas took place in 2019 and 2020, and activity should be comparable between 2019 and 2020 net of any exogenous shocks. Consequently, changes in program activity in 2020 can be interpreted as resulting from the disruptions due to the pandemic and transition to virtual means.

First, several program performance metrics are analyzed: number of scheduled visits, visit completion rates, time to completed visit, frequency of guidance provision (that is, the percentage of visits during which the nurse addressed families' needs through education and guidance), and community referral rates (that is, the percentage of visits during which the nurse offered a community referral to address needs in addition to providing education and guidance). Completion rates during the pandemic account for both the modified IHVs and the structured supportive calls.

Second, we report education/provision and community referral rates stratified by the caregiver's race and ethnicity and by the family's source of insurance. Race and ethnicity categories mirror the language used in the FC database: non-Hispanic white (thereafter white), non-Hispanic Black (thereafter Black), Hispanic, and non-Hispanic other. For source of insurance, we distinguish between families using private insurance and families using Medicaid or uninsured. Socio-demographic indicators are not available for families that did not complete the visit and we cannot comment on FC's population reach across specific subpopulations.

To analyze changes in program implementation during the COVID-19 pandemic, performance metrics are compared for two periods: March 1st-Nov. 30th, 2019 (prepandemic) and March 1st-Nov. 30th 2020 (post-pandemic onset). The cut-off in March reflects the shift towards state mandated closures (White House Communications, 2020) and the beginning of the FC transition to virtual means in 2020. As a sensitivity analysis, we introduced a cut-off in mid-March and obtained numerically and substantively
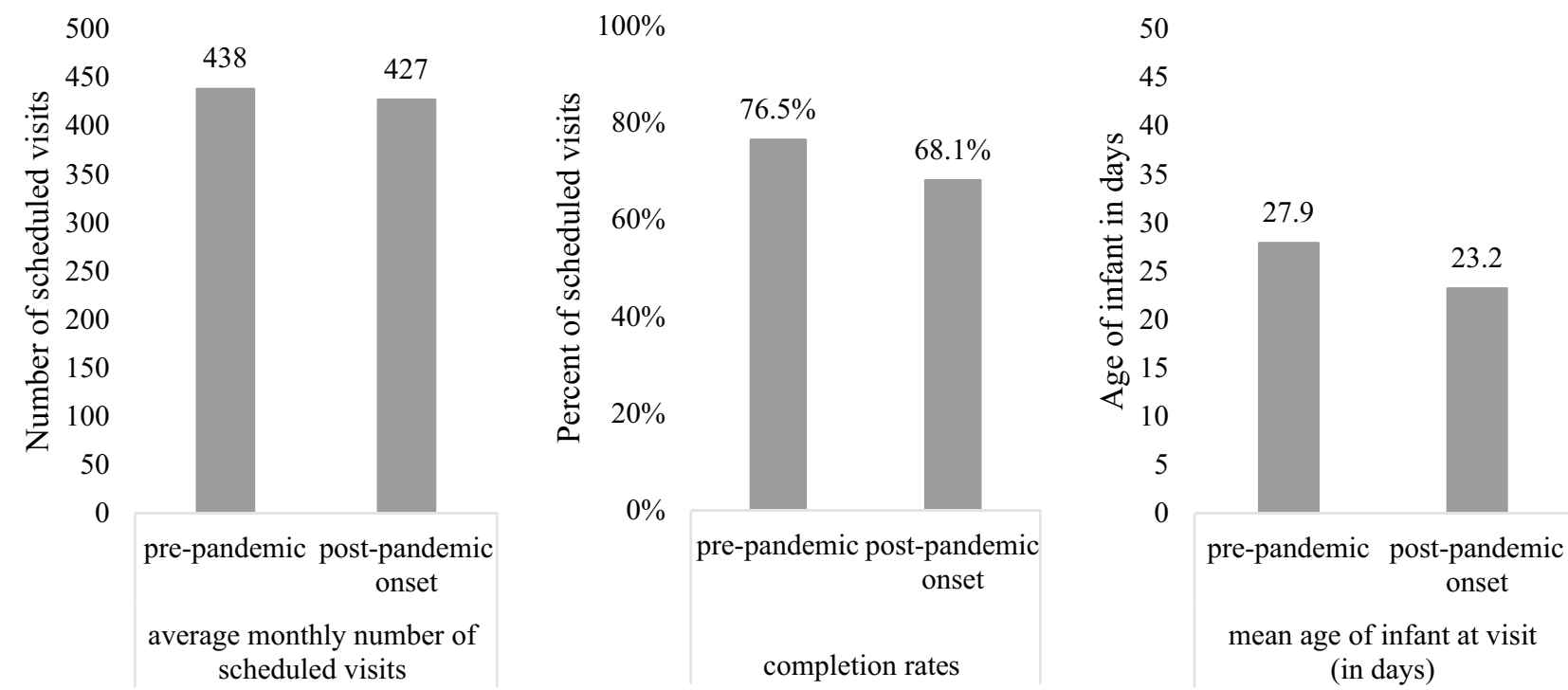

Fig. 1 Family Connects' program activity. Pre-pandemic data for March-Nov. 2019. Post-pandemic data for March-Nov. 2020. Metrics calculated using infant's date of birth to delineate analysis time period 
similar results. November was the last month for which the implementation data were available at the time of submission.

Last, to examine families' perceptions of the FC virtual visits, we use the COVID-19 Modification Survey conducted between May and November 2020. The Modification Survey is a short, 6-item, questionnaire distributed via phone call or e-mail approximately four weeks after the completed visit. The survey was designed as anonymous and not linked to other information about the family or FC visit, in order to accelerate IRB protocol approval and facilitate survey distribution. Families are asked whether the amount of contact with FC was satisfactory and how FC affected their feelings of social isolation and concerns about in-person medical care seeking. Across the five selected sites, 330 families who received a FC visit completed the survey (response rate $54.8 \%$ ). We present percentage distributions of answers for each relevant question item from the survey.

\section{Results}

Changes in post-pandemic FC activity are presented in Fig. 1 and Table 1 below. Pre-pandemic, on average 438 visits were scheduled monthly in the five analyzed FC sites compared to 427 visits post-pandemic onset, indicating a $2.5 \%$ decline in scheduling activity. Completion rates averaged at $76.5 \%$ pre-pandemic and declined by $10.9 \%$ to $68.1 \%$ post-pandemic onset. In the first nine months postpandemic onset, $78.9 \%$ of all virtual visits were completed as modified IHVs and $21.1 \%$ were completed as structured supportive calls.

The average age of the infant at the moment of the inperson visit pre-pandemic was 27.9 days. During the virtual delivery post-pandemic onset, the time to completed visit was shorter, with infants' mean age at visit equaling to 23.2 days. Modified IHVs were completed sooner post-birth (mean infant age of 22.6) than structured supportive calls (mean infant age of 25.7 days).
Table 1 Changes in Family Connects' program activity post-pandemic onset

\begin{tabular}{ll}
\hline Performance metric & March-November \\
& $2020 /$ March-November \\
& 2019 \\
\hline All families & \\
Average monthly number of scheduled visits & $-2.5 \%$ \\
Completion rates & $-10.9 \%$ \\
Education/guidance provision & $-0.3 \%$ \\
Community referral rates & $-12.3 \%$ \\
Mean time to visit completion & $-16.8 \%$ \\
Families of non-Hispanic Black caregivers & \\
Education/guidance provision & $-0.2 \%$ \\
Community referral rates & $-15.3 \%$ \\
Families of Hispanic caregivers & \\
Education/guidance provision & $-2.4 \%$ \\
Community referral rates & $-39.5 \%$ \\
Families of non-Hispanic white caregivers & \\
Education/guidance provision & $+0.4 \%$ \\
Community referral rates & $+8.7 \%$ \\
Families of non-Hispanic caregivers of all other races & \\
Education/guidance provision & $+0.1 \%$ \\
Community referral rates & $+16.3 \%$ \\
Families using Medicaid or uninsured & \\
Education/guidance provision & $-0.6 \%$ \\
Community referral rates & \\
Families using private health insurance & \\
Education/guidance provision & \\
Community referral rates & \\
\hline & \\
\hline
\end{tabular}

Family Connects' program implementation data, five mature and certified Family Connects sites. Scheduling activity and completion rates calculated using a sample of 7,791 scheduled visits and using infant's date of birth to delineate analysis time period. Education and referral activity metrics calculated using a sample of 5,112 completed visits (in-person IHVs and modified virtual IHVs) and using date of visit to define analysis time 


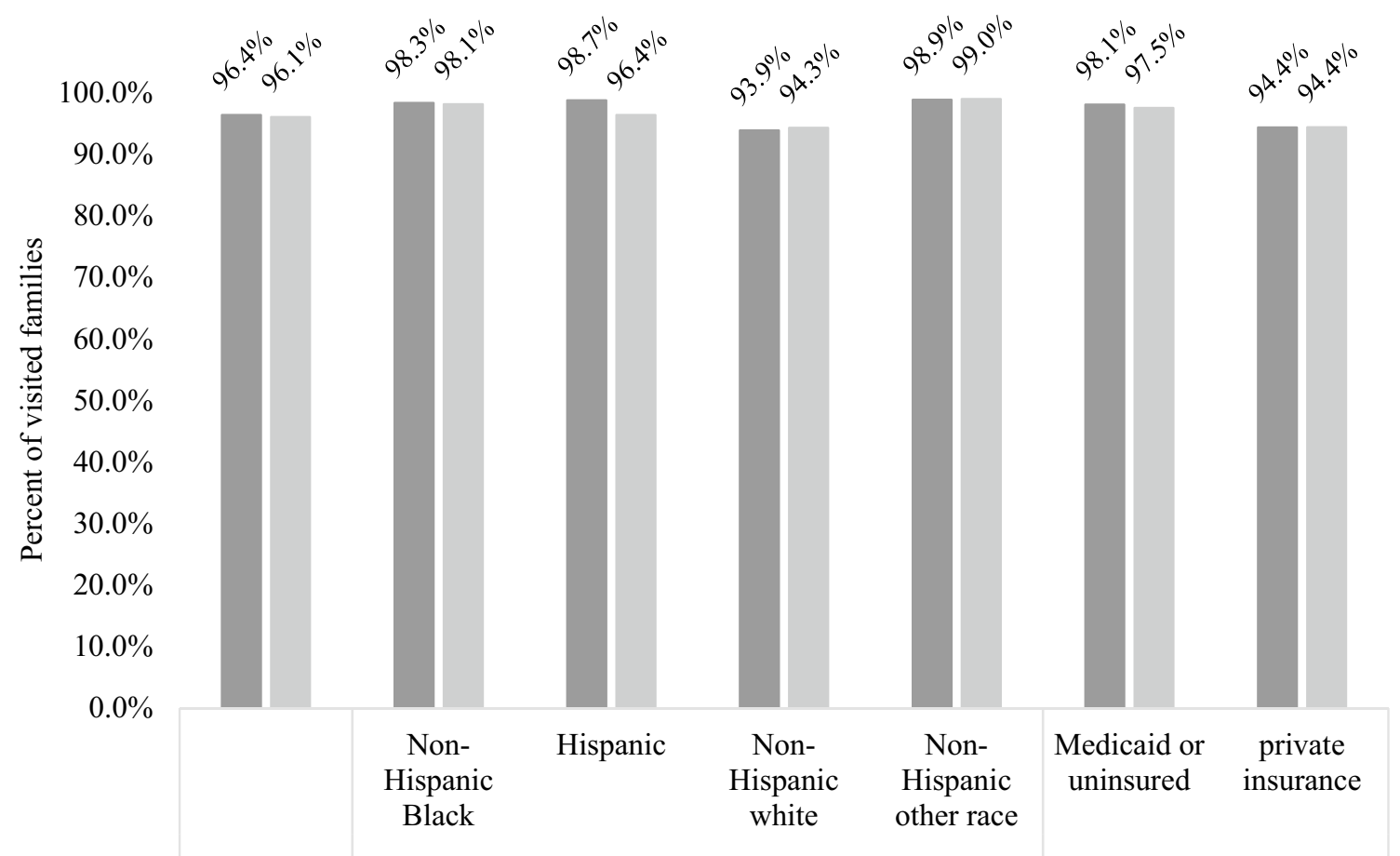

All families

By race/ethnicity

By source of insurance

Percent of families receiving education

$\square$ pre-pandemic post-pandemic onset

Fig. 2 Provision of education during FC visits. Pre-pandemic data for March-Nov. 2019. Post-pandemic data for March-Nov. 2020. Education provision calculated using a sample of 5112 completed visits (in-person IHVs and modified virtual IHVs) and using date of visit

Figure 2 represents frequency of education and guidance provision during the FC visit. Among families who completed traditional in-person IHVs before the pandemic, $96.4 \%$ of families received education and guidance during the in-person visit. In comparison $96.1 \%$ received these services during the modified virtual IHV post-pandemic onset. Further, across all four designated race/ethnicity subpopulations, the level of education provision was high prepandemic onset. On average, $98.3 \%$ of families with Black caregivers and $98.7 \%$ of families with Hispanic caregivers received guidance compared to $93.9 \%$ of families with white caregivers. After the pandemic onset, rates of education provision declined by $2.4 \%$ among Hispanic families but remained largely unchanged for all other groups (see also Table 1). Respectively $98.1 \%$ of families using Medicaid or uninsured and $94.4 \%$ of families using private insurance received education provision pre-pandemic. The post-pandemic onset values for education provision remained very similar, at $97.5 \%$ for families with Medicaid or uninsured and $94.4 \%$ for families with private insurance.

FC staff offered community referrals to $49.9 \%$ of visited families pre-pandemic onset and to $43.7 \%$ of to define analysis time. Due to data limitation, no information about education and referral activity is available for post-pandemic structured supportive calls

families post-pandemic onset, a decline of $12.3 \%$ (Fig. 3 and Table 1). We observed variation in referral rates across families of different racial and ethnic background before the pandemic. About $61.6 \%$ of families with Black caregivers were offered a referral, compared to $70.0 \%$ of families with Hispanic caregivers and $36.0 \%$ of families with white caregivers. Among families of Black and Hispanic caregivers, referral rates declined post-pandemic onset, by $15.3 \%$ and $39.5 \%$ respectively. In contrast, more families of white caregivers received community referrals post-pandemic onset, an increase of $8.7 \%$. Among families using Medicaid and uninsured families, $67.8 \%$ were offered a referral during the FC in-person visit pre-pandemic onset compared to $29.1 \%$ of families using private insurance. About $57.3 \%$ of Medicaid/ uninsured families were offered a referral once the pandemic started, a decline of $15.5 \%$. At the same time, the proportion offered a referral among families with private insurance increased by $3.5 \%$, to $30.1 \%$.

Based on data from the COVID-19 Modification Survey (Fig. 4), 89.0\% of families reported that the frequency of conversations with FC nurses was just right post-pandemic onset. Further, $65.1 \%$ of surveyed families reported that their 


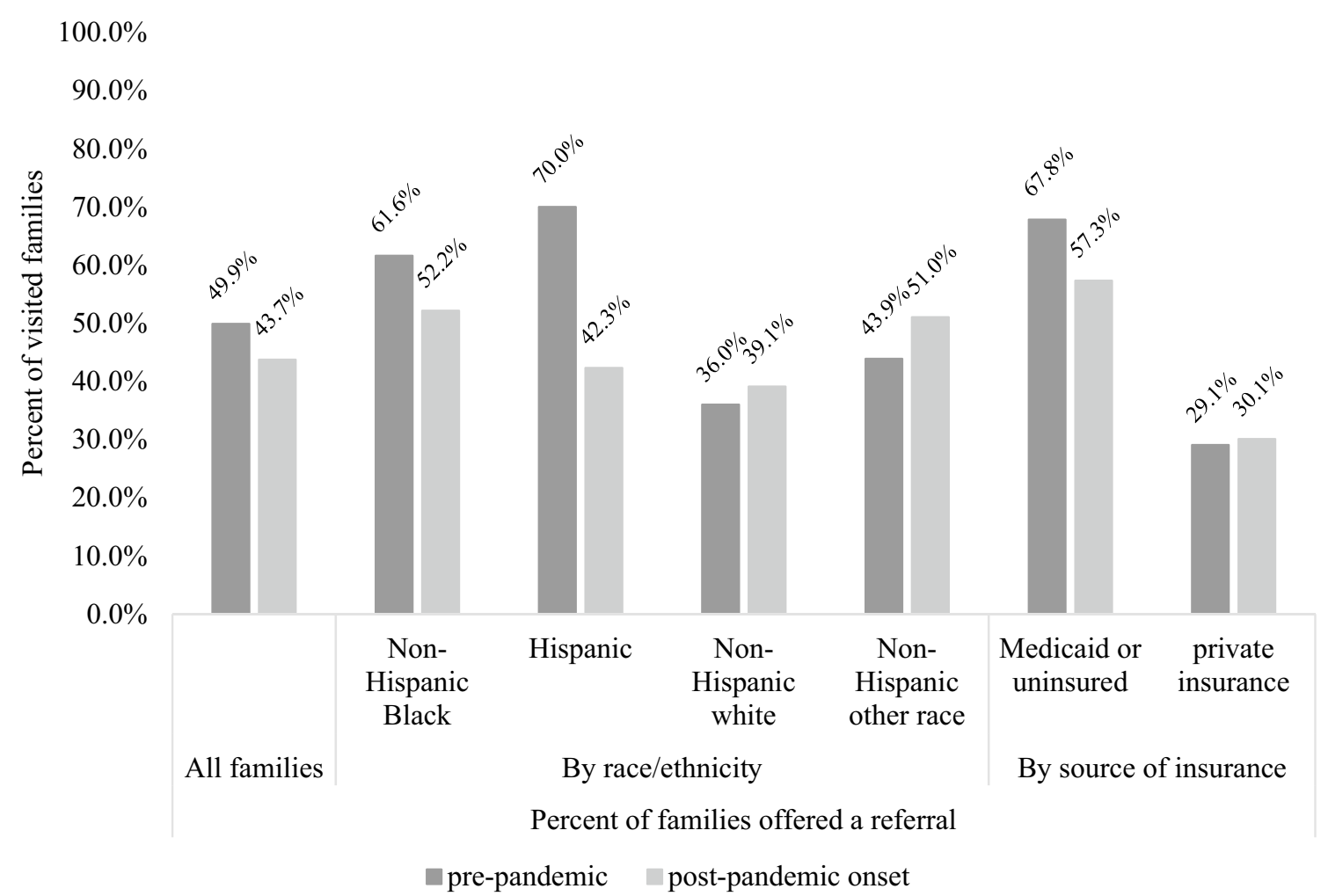

Fig. 3 Community referral rates during FC visits. Pre-pandemic data for March-Nov. 2019. Post-pandemic data for March-Nov. 2020. Referral rates calculated using a sample of 5112 completed visits (in-person IHVs and modified virtual IHVs) and using date of visit

feelings of isolation during the pandemic decreased because of their contact with the FC nurse. In addition, $61.6 \%$ of families reported being worried about seeking in-person routine medical care for themselves or their newborn infant during the pandemic. Among these families, $61.7 \%$ reported that their concerns declined because of their conversations with the FC nurse.

\section{Discussion}

Maternal, infant, and early childhood home visiting programs experienced a tremendous disruption in activities during the COVID-19 pandemic, a time when support for families with small children was extremely important. Data from Family Connects, a postpartum nurse home visiting intervention that transitioned to virtual delivery in March 2020, demonstrates that FC recruited comparable numbers of families into the program pre- versus post-pandemic onset. Completion rates declined slightly, by $10.9 \%$ postpandemic onset compared to pre-pandemic, but remained high at $68.1 \%$ of scheduled visits. Further, key components of the intervention, the provision of education and community referrals with respect to four key domains of family to define analysis time. Due to data limitation, no information about education and referral activity is available for post-pandemic structured supportive calls

well-being (health care, infant care, home safety, and parental well-being), were maintained at high levels during virtual delivery post-pandemic onset. FC staff provided education to $96.1 \%$ of the caregivers (compared to $96.4 \%$ pre-pandemic) and offered referrals for community agencies to $43.7 \%$ of caregivers (compared to $49.9 \%$ pre-pandemic). Finally, we report that families were satisfied with the level of communication with FC staff and reported decreased feelings of isolation and reduced concerns about seeking in-person services after the FC visit.

Taken together, these findings demonstrate the feasibility of transitioning a home visiting program to virtual means, meriting continued implementation and state support for home visiting interventions. We thus recommend that during the ongoing public health emergency, home visiting programs continue implementation with virtual modifications and that existing funding sources continue or grow. In addition, we suggest that the virtual home visiting protocol established during the COVID-19 pandemic can serve as a blueprint for virtual outreach in future emergency situations. During emergencies such as hurricane or wild fire evacuations, virtual services might provide necessary consultations and community connections for families. Similarly, virtual services can lead to expansion of the home visiting reach 


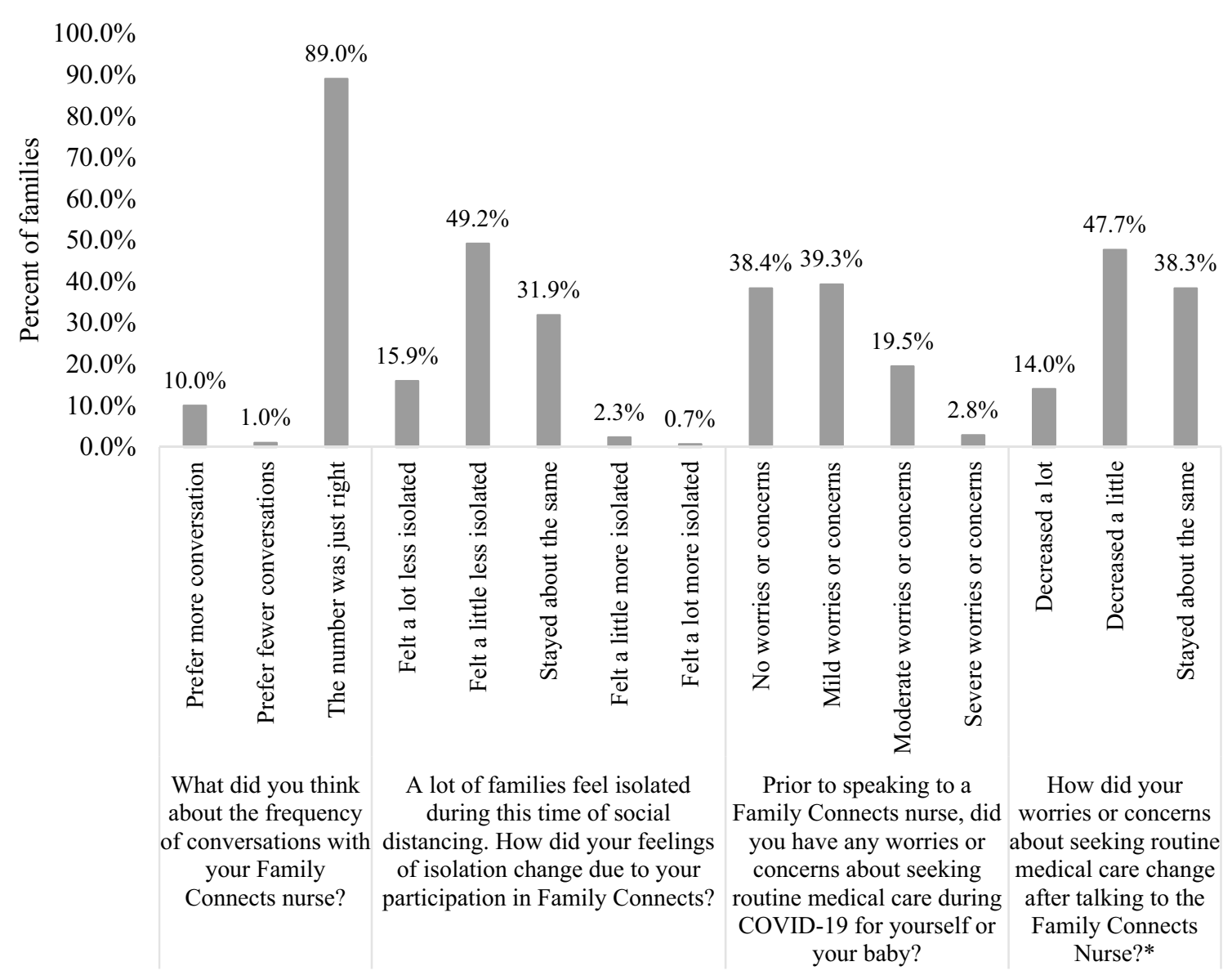

Fig. 4 Families' self-reported reception of Family Connects' visits during the COVID-19 pandemic from the COVID-19 Modification Survey $(\mathrm{N}=330)$. *Question asked only among respondents who

by offering virtual visits to families reluctant to welcome a nurse into their home or to families in remote areas. In summary, even though the COVID-19 imposed modifications to home visiting might be temporary, we expect the option of virtual delivery to remain within the portfolio of services offered by home visiting programs.

While we conclude that the transition to virtual means of home visiting delivery during COVID-19 was successful for FC, we recognize that in-person and in-home observations are evidence-based components of home visiting interventions, demonstrated to improve family well-being. The impact of virtual delivery (that is, whether this mode is associated with positive outcomes for families) is unknown. Consequently, a rigorous evaluation of the impact of virtual delivery on health outcomes, e.g., infant development, parental mental health or parent-infant relationship, is necessary. We also suggest future studies for FC about potential obstacles to virtual visit completion to remedy declining follow-through rates. Potential reasons for the small decline in program completion could include poor internet/cell data access, families' lack of time to complete visits, or staffing reported mild to severe worries about seeking routine medical care during COVID-19 for themselves or their baby

shortages with home visiting nurses delegated to COVID-19 relief efforts.

Additionally, while we document declines in community referral rates, we cannot explain why these declines are observed. On one side, a decline in referrals might indicate that, during the pandemic, FC nurses recognize the difficulty families have in attending community services (because the services have closed or the family is reluctant to reach out) and so the nurses are taking on the task of addressing the need during the visit rather than connecting the family to a community agency. On the other hand, a decline in provision of education and referrals might indicate that some needs are not being reported adequately by the parents or assessed fully by the nurses, whilst before, needs would have been observed directly by the nurse visiting the home and performing physical examinations. Future research should address these unanswered questions.

As home visiting programs continue services virtually and consider future changes to the intervention protocols, careful consideration should be devoted to issues of equal access to the interventions and their benefits for all 
families within participating communities. Findings in this paper show that while the community connections of affluent families and white families increased during the pandemic, these linkages weakened for low-income families and families of color. These noteworthy differences in program activity might reflect lack of access to services necessary for virtual home visiting, such as broadband internet, but also disproportionate impact of the pandemic on these communities. Thus, a priority of future research ought to be a critical examination of the reasons behind lower community referrals among minority and low-income families and an investigation of potential community alignment solutions to improve connectedness among historically marginalized families.

Acknowledgements We acknowledge the contributions of many staff members and community leaders in implementing Family Connects and its evaluation. The authors thank Phil Nousak for his assistance with data management and 4 anonymous reviewers for their helpful comments on previous versions of the manuscript.

Author Contributions All Authors participated in study conception and design. Dr. Rybińska carried out the program activity analyses and interpreted the data, drafted the initial manuscript, and reviewed and revised the manuscript. Drs. Best, Dodge, and Goodman participated in interpretation of data and reviewed and revised the manuscript. Ms. Weindling carried out analyses for the COVID19 Modification Survey and reviewed and revised the manuscript. All authors approved the final manuscript as submitted and agree to be accountable for all aspects of the work.

Funding Funding for this research was provided by The Duke Endowment (TDE \#20-01-SGO) and the R01HD069981 grant from the Eunice Kennedy Shiver National Institute for Child Health and Human Development.

\section{Declarations}

Conflicts of interest Drs. Best, Dodge, and Goodman acknowledge participation in Family Connects model dissemination. As the founder of Family Connects, Dr. Dodge provides periodic, in-kind consultation to sites implementing Family Connects. As director of research for Family Connects, Dr. Goodman supports local evaluation efforts at some dissemination sites. As medical director and national director of implementation for Family Connects, Dr. Best oversees site training and advises on local site clinical implementation work for dissemination sites. The other authors declare no conflict of interest.

Ethics Approval Research protocols for this study were approved by the Duke University Health System's IRB (Protocol \#00105777) and the Duke University Campus IRB (Protocol \#2021-0197).

Consent to Participate Not applicable.

Consent for Publication Not applicable.

Availability of Data and Material Data used in this research are deidentified participant data. Data are collected and managed by the Center for Child \& Family Health, a community non-profit in Durham, NC, that serves as the national training and dissemination hub for Family Connects program. Data are not publicly available.
Code Availability Code is not publicly available per study protocol guidelines.

\section{References}

Administration for Children and Families. (2020). Early childhood home visiting models: Reviewing evidence of effectiveness. Mathematica. Retrieved from October 6, 2021https://www.mathematica.org/publications/early-childhood-home-visiting-models-revie wing-evidence-of-effectiveness

Alonso-Marsden, S., Dodge, K. A., O’Donnell, K. J., Murphy, R. A., Sato, J., \& Christopoulos, C. (2013). Family risk as a predictor of initial engagement and follow-through in a universal nurse home visiting program to prevent child maltreatment. Child Abuse and Neglect. https://doi.org/10.1016/j.chiabu.2013.03.012

Dodge, K. A., Goodman, W. B., Bai, Y., O’Donnell, K. J., \& Murphy, R. A. (2019). Effect of a community agency-administered nurse home visitation program on program use and maternal and infant health outcomes: A randomized clinical trial. JAMA Network Open. https://doi.org/10.1001/jamanetworkopen.2019.14522

Dodge, K. A., Goodman, W. B., Murphy, R. A., O'Donnell, K., Sato, J., \& Guptill, S. (2014). Implementation and randomized controlled trial evaluation of universal postnatal nurse home visiting. American Journal of Public Health, 104(S1), S136-S143.

Dodge, K. A., Goodman, W. B., Bai, Y., O’Donnell, K. J., \& Sato, J. (2013). Randomized controlled trial of universal postnatal nurse home visiting: Impact on emergency care. Pediatrics. https://doi. org/10.1542/peds.2013-1021M

Cluver, L., Lachman, J. M., Sherr, L., Wessels, I., Kurg, E., Rakotomalala, S., et al. (2020). Parenting in a time of COVID-19. The Lancet. https://doi.org/10.1016/S0140-6736(20)30736-4

Goodman, W. B., Dodge, K. A., Bai, Y., Murphy, R. A., \& O’Donnell, K. (2021). Effect of a universal postpartum nurse home visiting program on child maltreatment and emergency medical care at 5 years of age: A randomized clinical trial. JAMA Network Open. https://doi.org/10.1001/jamanetworkopen.2021.16024

Goodman, W. B., Dodge, K. A., Bai, Y., O’Donnell, K. J., \& Murphy, R. A. (2019). Randomized controlled trial of Family Connects: Effects on child emergency medical care from birth to 24 months. Developmental Psychology. https://doi.org/10.1017/S095457941 9000889

Family Connects International. (2019). Retrieved August 27, 2020, from https://familyconnects.org

Family Connects International. (2019b). Family Connects locations. Retrieved August 27, 2020, from https://familyconnects.org/family-connects-model/family-connects-sites

Health Resources \& Services Administration. (2020). Maternal, infant, and early childhood home visiting program. HRSA maternal and child health. Retrieved August 27, 2020, from https://mchb.hrsa. gov/sites/default/files/mchb/MaternalChildHealthInitiatives/ HomeVisiting/pdf/home-visiting-infographic.pdf

Health Resources and Services Administration \& Centers for Medicare and Medicaid Services. (2016). Coverage of maternal, infant, and early childhood home visiting services. Retrieved July 31, 2018, from https://www.medicaid.gov/federal-policy-guidance/downl oads/CIB-03-02-16.pdf

Kirby, T. (2020). Evidence mounts on the disproportionate effect of COVID-19 on ethnic minorities. The Lancet Respiratory Medicine, 8(6), 547-548.

Marshall, J., Kihlström, L., Buro, A., Chandran, V., Prieto, C., SteinElger, R., et al. (2020). Statewide implementation of virtual 
perinatal home visiting during COVID-19. Maternal and Child Health Journal. https://doi.org/10.1007/s10995-020-02982-8

Raifman, M., \& Raifman, J. (2020). Disparities in the population at risk of severe illness from COVID-19 by race/ethnicity and income. American Journal of Preventive Medicine. https://doi.org/10. 1016/j.amepre.2020.04.003

Self-Brown, S., Reuben, K., Perry, E. W., Bullinger, L. R., Osborne, M. C., Bielecki, J., et al. (2020). The impact of COVID-19 on the delivery of an evidence-based child maltreatment prevention program: Understanding the perspectives of SafeCare ${ }^{\circledR}$ providers. Journal of Family Violence. https://doi.org/10.1007/ s10896-020-00217-6

White House Communications. (2020). Proclamation on declaring a national emergency concerning the novel coronavirus disease (COVID-19) outbreak. Whitehouse.gov. Retrieved August 27.
2020, from https://www.whitehouse.gov/presidential-actions/procl amation-declaring-national-emergency-concerning-novel-coron avirus-disease-covid-19-outbreak

Williams, K., Ruiz, F., Hernandez, F., \& Hancock, M. (2020). Home visiting: A lifeline for families during the COVID-19 pandemic. Archives of Psychiatric Nursing. https://doi.org/10.1016/j.apnu. 2020.10 .013

Zero to Three. (2020). States modify home visiting services in response to COVID-19. Retrieved December 23, 2020, from https://www. zerotothree.org/resources/3315-states-modify-home-visiting-servi ces-in-response-to-covid-19

Publisher's Note Springer Nature remains neutral with regard to jurisdictional claims in published maps and institutional affiliations. 